\title{
The false positive troponin results: case studies of analytical interference
}

\author{
Authors: Saerrah Murryam, ${ }^{A}$ Paul Cook $^{B}$ and Sebastien Ellis ${ }^{C}$
}

Cardiac troponin I and T are particularly sensitive and specific markers for cardiomyocyte damage. Myocardial injury can occur due to a discrepancy between oxygen supply and demand (eg coronary artery occlusion and arrhythmias), other cardiac causes (eg pericarditis, myocarditis, cardiac surgery, cardioversion etc) or systemic conditions (eg sepsis, stroke and chronic renal disease). The latest European Society of Cardiology guidelines help to guide clinicians through these different causes. Occasionally troponin concentrations may not fit the clinical presentation and, therefore, other aetiologies should be considered. An under-appreciated basis of a high troponin concentration is a false positive result, which can be attributable to analytical interference from components in the patient's blood. Uncovering this interference can be pivotal to avoid unnecessary and potentially harmful investigations or treatment for patients. We present two cases of false positive troponin results caused by analytical interference. The normal reference range for the assay (Access; Beckman Coulter, High Wycombe, UK) used at our organisation is $0-18 \mathrm{ng} / \mathrm{L}$.

KEYWORDS: troponin, heterophile antibody, analytical interference

DOI: $10.7861 /$ clinmed.2021-0633

\section{Introduction}

Troponin I and T are expressed solely within cardiac muscle and are released into the bloodstream following cardiomyocyte death. These biomarkers are commonly used for the diagnosis of acute myocardial infarction. A false positive result should be considered where there is a clear discrepancy in the clinical presentation of a patient. Antibody interference in immunoassays (whereby a substance is measured using antibodies) is a well-recognised phenomenon and can affect all analytes and all assays regardless of manufacturer. ${ }^{1}$

Authors: ${ }^{A}$ internal medical trainee year 2, University Hospital Southampton NHS Foundation Trust, Southampton, UK; B biochemistry consultant, University Hospital Southampton NHS Foundation Trust,

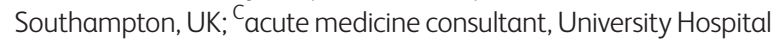
Southampton NHS Foundation Trust, Southampton, UK

\section{Case presentations}

\section{Case 1}

An ordinarily fit and well 38-year-old man presented with a 24-hour history of sharp central chest pain while doing household chores. He described the pain being worse on sternal palpation and inspiration. He had no other associated symptoms. He had no past medical history, drug history or relevant family history. His serial electrocardiography (ECG) revealed normal sinus rhythm with no ischaemic changes. He had a normal chest $X$-ray. His troponin concentration in the emergency department was $66 \mathrm{ng} / \mathrm{L}$ then $75 \mathrm{ng} / \mathrm{L}$ (with a negative D-dimer). He then had transthoracic echocardiography (TTE) showing no structural abnormalities. He was initially treated for myopericarditis with ibuprofen and colchicine after discussion with cardiology. His blood samples were sent for analysis on a different analytical platform to exclude analytical interference, on retesting, the troponin result was normal. He was contacted to discontinue medications for myopericarditis. The eventual diagnosis was musculoskeletal chest pain.

\section{Case 2}

A 58-year-old man with a background of a previous myocardial infarction (MI) and coronary artery bypass surgery, type 2 diabetes mellitus, hypercholesterolaemia and obesity presented on three separate occasions with chest pain. During the first episode, he was admitted under cardiology with a troponin concentration of $334 \mathrm{ng} / \mathrm{L}$ and subsequently $233 \mathrm{ng} / \mathrm{L}$. His ECGs showed no ischaemic change and TTE was normal. Due to his elevated troponin results, a cardiac magnetic resonance imaging was arranged that showed no acute myocardial injury and no inducible ischaemia with a normal left ventricular ejection fraction. Nevertheless, he was diagnosed and treated for an MI with conservative management and discharged home.

He re-presented 3 months later to the acute medical unit with chest pain of a similar nature. His troponin concentrations were again noted to be statically raised (183-210 ng/L). At this point he was rediscussed with cardiologists who requested an outpatient computed tomography coronary angiography, and he was discharged. This demonstrated patent grafts from his bypass with no evidence of stenosis. Troponin samples during this admission were sent for further analysis on a different analytical platform and were normal. 


\begin{tabular}{|c|c|}
\hline False positive causes & $\begin{array}{l}\text { True raise in troponin not } \\
\text { related to myocardial infarction }\end{array}$ \\
\hline High alkaline phosphatase & Myocarditis/pericarditis \\
\hline Fibrin clots & Pulmonary embolism \\
\hline Haemolysis & Congestive heart failure \\
\hline $\begin{array}{l}\text { Heterophile antibody } \\
\text { interference }\end{array}$ & Arrhythmias \\
\hline $\begin{array}{l}\text { Microparticles in specimen } \\
\text { sample }\end{array}$ & Chronic kidney disease \\
\hline Immunocomplex formation & Cardiac surgery \\
\hline Rheumatoid factor & $\begin{array}{l}\text { Cardiac procedures: ablation, } \\
\text { cardioversion and primary } \\
\text { percutaneous coronary } \\
\text { intervention }\end{array}$ \\
\hline Auto-antibodies & Critical illness \\
\hline \multirow[t]{2}{*}{$\begin{array}{l}\text { Interfering endogenous } \\
\text { components in blood eg } \\
\text { bilirubin, haemoglobin and } \\
\text { lipaemia }\end{array}$} & Severe anaemia \\
\hline & Inflammatory conditions \\
\hline
\end{tabular}

He re-presented once more under the cardiology team. Although he continued to have unremarkable ECG and TTE findings, he was conservatively managed for an MI again as his troponin concentrations were 202-242 ng/L. Results of further troponin analysis showing false positive results were not yet available to clinicians.

\section{Discussion}

Antibody interference in immunoassays is a well-recognised phenomenon and can affect all analytes and assays regardless of manufacturer. The key to detection is clinical, ie the result does not fit the clinical picture and, in the case of troponin, a static nondynamic concentration. It is the rise and fall of troponin levels that is key when diagnosing myocardial ischaemia.

Interfering antibodies can include heterophilic antibodies that have multi-specificity for antigens and include proteins such as rheumatoid factor, anti-animal antibodies (the most well documented being human anti-mouse antibodies that bind to the animal-derived antibodies used in immunoassays), or autoantibodies formed against one of the body's own components. ${ }^{2}$ Table 1 summarises some of these causes of false positive troponin results. ${ }^{2}$ The exact effect of the interference will depend on the type of assay used and the site where the antibody binds to the analyte. Interference may lead to either falsely elevated or decreased values.

Heterophilic antibodies are a group of poorly defined endogenous antibodies that react with many different molecules. They are characterised by substantial non-specificity, developed in response to no clear immunogen. These antibodies can be associated with autoimmune and other inflammatory diseases but are also present to a lesser extent in apparently healthy individuals. This non-specificity explains why one may get a false positive on occasion with one assay that is not evident on a different analytical platform. ${ }^{2}$

Our cases demonstrate the importance of interpreting a troponin concentration in the context of each patient's presentation as well as other clinical results. In both patients, the statically raised troponin concentrations did not go hand in hand with their presentations. Misinterpretation can result in incorrect diagnoses, and unnecessary investigations and treatments. ${ }^{3}$

\section{Key points}

> Troponin I and T are highly sensitive and specific as they are expressed solely within the myocardium.

$>$ Once other non-cardiovascular conditions (such as chronic kidney disease and sepsis) are ruled out, a false positive result should be suspected (particularly in statically raised results that do not fit the clinical picture).

$>$ Following clinical correlation, consider sending samples for analysis on a different immunoassay testing platform to objectively demonstrate a false positive result: once identified, this can significantly impact future patient management.

$>$ Our cases show the importance of interpreting troponin concentrations in the context of a patient's clinical state and other investigations, such as serial ECGs.

\section{References}

1 Lakusic N, Sopek Merkas I, Lucinger D, Mahovic D. Heterophile antibodies, false-positive troponin, and acute coronary syndrome: a case report indicating a pitfall in clinical practice. Eur Heart ] Case Rep 2021;5:ytab018.

2 Mair J, Lindahl B, Müller C et al. What to do when you question cardiac troponin values. Eur Heart ] Acute Cardiovasc Care 2018:7:577-86.

3 Lum G, Solarz DE, Farney L. False positive cardiac troponin results in patients without acute myocardial infarction. Laboratory Medicine 2006;37:546-50.

Address for correspondence: Dr Saerrah Murryam, Southampton General Hospital, University Hospital Southampton NHS Foundation Trust, Tremona Road, Southampton, Hampshire S016 6YD, UK. Email: saerrah.murryam@uhs.nhs.uk 